\title{
miRNA and mRNA expression profiles in gastric cancer patients and the relationship with circRNA
}

\author{
F. JIANG ${ }^{1,2, *}$, X. B. SHEN ${ }^{1,2, \#, *}$ \\ ${ }^{1}$ Key Laboratory of Environmental Medical Engineering and Education Ministry, Nanjing Public Health College, Southeast University, Nanjing, \\ China; ${ }^{2}$ Department of Preventive Medicine, Nanjing Public Health College, Southeast University, Nanjing, China
}

${ }^{*}$ Correspondence: xb.shen@seu.edu.cn

"Contributed equally to this work.

Received December 11, 2018 / Accepted March 28, 2019

\begin{abstract}
Gastric cancer (GC) is a malignant tumor that affects individuals worldwide, and miRNA and mRNA are closely connected to this disease. However, it is still unclear how these molecules affect GC and whether their effects are associated with circRNA in GC patients. Therefore, we obtained the miRNA, mRNA and circRNA expression profiles of GC patients from the GEO database. For comparison, shared miRNAs and mRNAs from the results of microarrays were annotated by gene ontology (GO) and pathway analysis. We also identified mRNAs that were targeted by miRNA through TargetScan 7.2 and circRNAs that were targeted by miRNA through CircInteractome. A comprehensive analysis of the microarray results revealed 72 shared miRNAs, and the expression profiles of 6 miRNAs were significantly different between the tumor and control groups (the absolute value of fold change $>2, \mathrm{p}<0.05$ ). Hsa-miR-1, hsa-miR-142-3p, hsa-miR-95, hsa-miR-133a and hsa-miR-181d were upregulated in GC, whereas hsa-miR-375 was downregulated. The analysis results also revealed 1201 shared mRNAs and 27 mRNAs by microarray and TargetScan. Pathway analysis demonstrated that the Glypican pathway, Proteoglycan syndecan-mediated signaling events, Glypican 1 network and PAR1-mediated thrombin signaling events played important roles. GO analysis revealed significant enrichment in the three terms cellular component, molecular function and biological process, suggesting that organelles, enzyme binding, RNA-binding and nitrogen metabolism may have a strong relationship in GC. The increase in PAX6 in GC may be related to hsa-miR-375. Three circRNAs, hsa_circ_0001658, hsa_circ_0004928 and hsa_circ_0000376, were then found to be significantly differentially expressed between GC and normal tissues (the absolute value of fold change $>2, \mathrm{p}<0.05$ ). In conclusion, the circ0001658/ circ0004928/circ0000376-miR-375-PAX6 axis may represent a new regulatory network that should be further investigated, and the results of this study provide a better understanding of GC.
\end{abstract}

Key words: gastric cancer, miRNAs, mRNAs, circRNAs, GEO

Gastric cancer (GC) is one of the most serious malignant tumors worldwide, with the fifth highest incidence rate and third highest mortality rate [1]. Moreover, more than half of these cases occur in Eastern Asia, Central and Eastern Europe, and Central and South America, especially Japan and China [2]. Although there are some steady decreases in the incidence rates and mortality of GC with improved nutrition and anti-HP antibody use, this disease still poses a formidable threat to human health, as GC patients typically have a poor prognosis [3]. The five-year survival rate of patients with late-stage gastric cancer is still very low, which may be because high specificity and high sensitivity biomarkers for early detection are still lacking [4]. Clearly, the molecular mechanisms of gastric cancer remain unclear. Although the tumor markers CA199, CEA, and CA724 can be used for the detection of gastric cancer, they have low sensitivity and specificity, especially for late-stage gastric cancer patients [5]. Thus, there is an urgent need to discover novel molecular biomarkers to increase the detection rate of gastric cancer.

MiRNAs are short, single-stranded RNA molecules that can regulate gene expression [6] and even protein expression [7]. miRNAs bind to mRNA at the 3'-untranslated region (UTR) and cause the downregulation of protein coding genes in the cytoplasm. Alternatively, miRNAs can also reduce the expression of mRNA-targeted proteins through increased mRNA degradation [8]. It is not surprising that the expressions of certain miRNAs are highly related to cancers [9]. For example, miR-21 is related to lung cancer [10] and colon cancer [11]; miR-133 is highly related to breast cancer [12]; 
miR-200c is related to ovarian cancer [13]; and hsa-miR-375 and hsa-miR-142-5p are related to gastric cancer [14].

CircRNAs are a special class of endogenous noncoding RNAs generated from splicing events or back-splicing events via exons, introns or exon-intron circularization [15]. The special back-splicing structure of circRNAs is different from linear RNA, which confers a variety of properties to circRNAs that have been validated only recently [16]. Many studies have shown that circRNA can act as a molecular sponge of miRNA to regulate gene expression, encode some proteins and regulate various biological processes, such as proliferation, migration and invasion [17]. Studies have also found that the expression of circRNAs was dysregulated in multiple types of cancer cell lines, which correlated with certain clinical outcomes, suggesting a potential biomarker role for circRNAs in tumor progression. Therefore, circRNAs may potentially serve as required novel biomarkers for gastric cancer [18].

Therefore, our goal is to identify miRNA and mRNA expression profiles in gastric cancer patients and examine the relationship with circRNA in order to provide basic information about gastric cancer and a foundation for further study.

\section{Materials and methods}

Sample collection. For this study, we obtained the expression profiles of miRNAs, mRNAs and circRNAs of gastric cancer patients in GEO, all through microarray in Homo sapiens. The patients were from domestic and foreign hospitals. Finally, we obtained data from 4 miRNA microarrays (GSE93415, GSE23739, GSE28700, GSE26645), 4 mRNA microarrays (GSE2685, GSE103236, GSE54129, GSE79973) and 1 circRNA microarray (GSE78092).

Differentially expressed RNAs. GEO2R (http://www. ncbi.nlm.nih.gov/geo/geo2r), an online analysis tool, was used to compare the differentially expressed RNAs of selected GEO microarrays in our study. The adjusted P values were used to correct for the occurrence of false positive results via the Benjamini and Hochberg false discovery rate method. Aiming to determine the differential expression of RNAs, we used the following criterion: the absolute value of fold change more than 2 and $P$ value less than 0.05 .

GO and pathway analysis. The Gene Ontology (GO) project, which is the most comprehensive resource, was used to acquire the functions of genes and gene products [19], which included the three categories cellular component, molecular function and biological process by FunRich v3 [20]. We also performed miRNA pathway analysis, which is an effective method, to determine the underlying biological functions of the differentially expressed miRNAs between gastric cancer and normal tissues using FunRich v3. The gene numbers and corrected $P$ values, which are the result of the hypergeometric test to correct for the occurrence of false positive results via the Benjamini and Hochberg false discovery rate method, were used in the analysis of GO terms and the biological pathway. A p-value less than 0.05 was considered significant.

The relationship between miRNA-mRNA and miRNAcircRNA. After examining the miRNA expression in gastric cancer and normal tissues (the absolute value of fold change $>2, \mathrm{p}<0.05$ ), which has also been studied by some investigators, we identified the mRNAs targeted by miRNA through TargetScan 7.2. Then, we identified the mRNAs that correlated with miRNA expression in gastric cancer through Vann. We also performed pathway analysis of the differential expression of mRNAs using DAVID Bioinformatics.

Similarly, we acquired the circRNA targeted by miRNA through CircInteractome. The 3 expressing obvious differently circRNAs were also annotated through circBase.

\section{Results}

Differential expression profiles of miRNAs in gastric cancer. The results of 4 miRNA microarrays are shown in Table S1.0. GSE93415 has 20 pairs of samples; GSE23739 has 40 pairs of samples; GSE28700 has 22 pairs of samples; and GSE26645 has 4 pairs of samples. There were 939, 795, 534 and 939 miRNAs differentially expressed between GC and normal tissues, respectively. A Venn plot was then used to identify microarray the shared miRNAs, and 72 shared miRNAs were found (Figure 1A, Table S1.1). Because the first detection time (2017) was relatively recent, there were many more tumor and adjacent tumor tissue samples available. Therefore, we performed further analysis of shared miRNAs with the first microarray dataset (Tables S1.2 and S1.3).

In terms of miRNA expression data in the hierarchical clustering map, we can separate the GC patients from controls (Figure 1B). Volcano plot analysis was then applied to examine the differentially expressed miRNAs between the two groups in the first microarray dataset. With an absolute value of fold change of more than 2 and a p-value of less than 0.05 as the cut off, we found that hsa-miR-1 $(\log \mathrm{FC}=1.6699, \mathrm{p}=2.69 \mathrm{E}-03)$, hsa-miR-142-3p $(\log \mathrm{FC}=1.5088, \mathrm{p}=2.66 \mathrm{E}-03)$, hsa-miR-95 $(\log \mathrm{FC}=1.42215$, $\mathrm{p}=1.06 \mathrm{E}-04)$, hsa-miR-133a $(\log \mathrm{FC}=1.0509, \mathrm{p}=3.44 \mathrm{E}-03)$ and hsa-miR-181d $(\log \mathrm{FC}=1.02855, \mathrm{p}=1.45 \mathrm{E}-08)$ were upregulated in GC, and hsa-miR-375 (logFC=-1.06525, $\mathrm{p}=3.30 \mathrm{E}-03)$ was downregulated in GC patients compared with controls (Figure 1C).

After reviewing the relevant literature, we found many studies on hsa-miR-375 in GC [14] and other diseases [21-25]. Therefore, we selected the downregulated miRNA hsa-miR-375 as our study subject. To evaluate the enrichment of hsa-miR-375 in the categories as cellular component, molecular function and biological process, GO analysis was performed (Figures S1A-C). Cellular component analysis revealed that hsa-miR-375 mainly existed in the nucleus and cytoplasm (Figure S1A). Molecular function analysis showed that hsa-mir-375 was active in transcription factor activity, transporter activity and RNA binding (Figure S1B). 
A

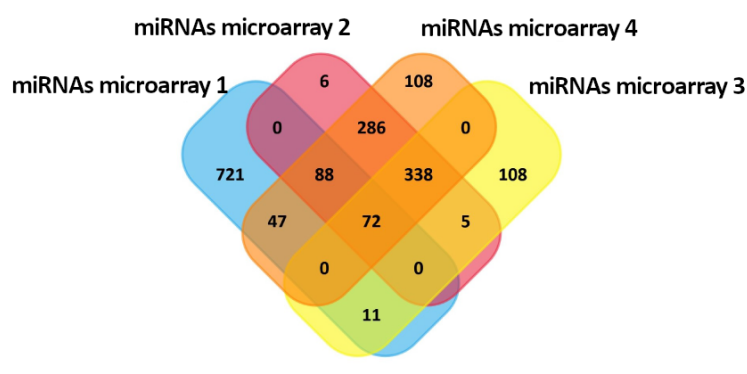

C

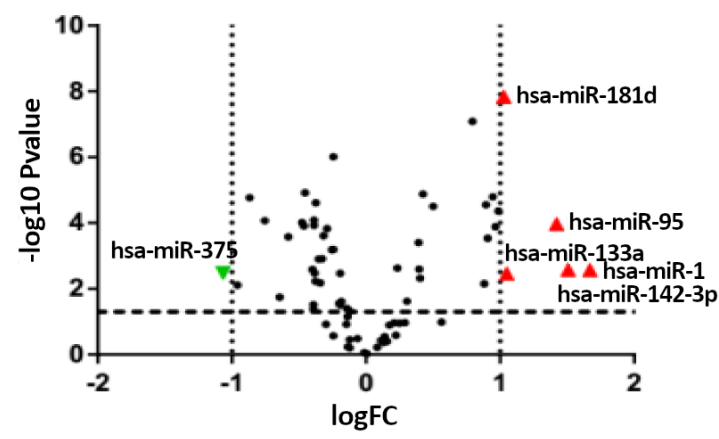

B

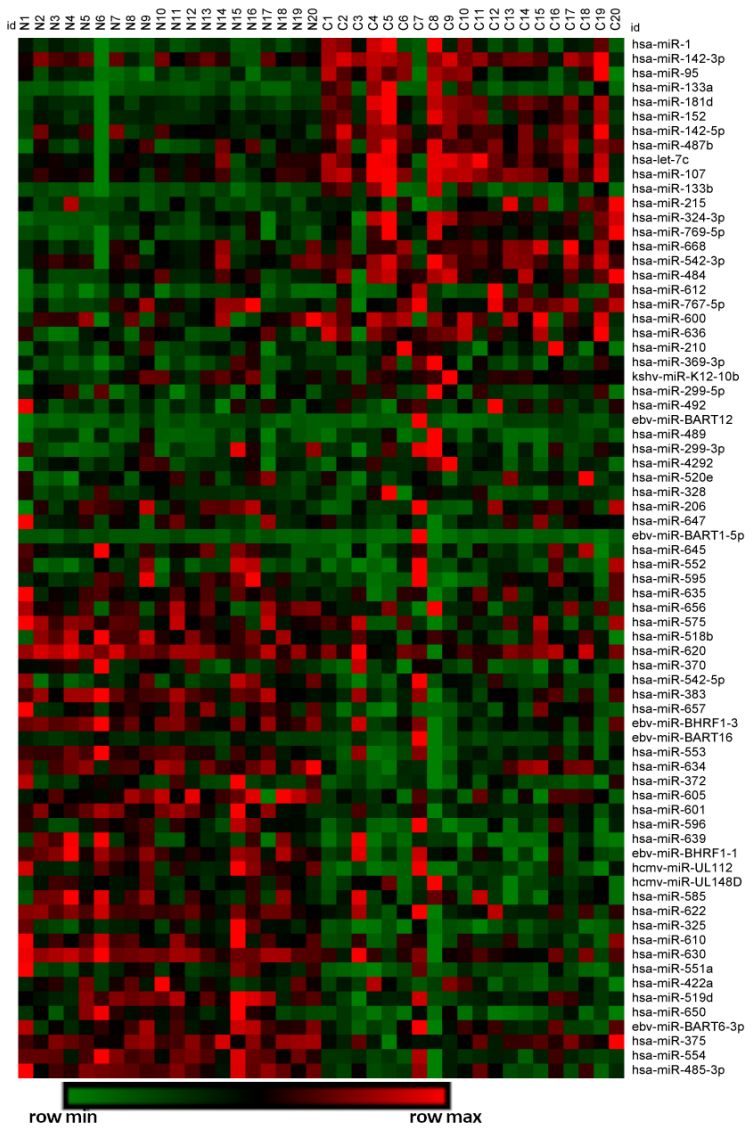

Figure 1. Expression profiles of miRNAs in gastric cancer. A) The number of miRNAs expressed between gastric cancer and normal tissues in the 4 microarray datasets, with 72 shared miRNAs. B) Hierarchical clustering analysis revealed 72 miRNAs with different expression profiles between GC (C1-C20) and adjacent tissues (N1-N20). The red and green shades represent expression levels above and below the relationship expression among all samples, respectively (row $\min$ is 0 , row $\max$ is 1 ). C) The differentially expressed miRNAs between gastric cancer and normal tissues in the volcano plot. The vertical lines correspond to 2 -fold upregulation or downregulation, and the horizontal lines represent $\mathrm{p}=0.05$. The red points highlight the upregulated miRNAs and the green points reflect the downregulated miRNAs.

Regulation of nucleobase, nucleoside, nucleotide and nucleic acid metabolism and transport were the two main biological processes (Figure S1C). Subsequent pathway analysis showed that hsa-miR-375 was involved in the glypican pathway, regulation of CDC42 activity, CDC42 signaling, developmental biology, Wnt signaling pathway, glypican 3 network, etc. (Figure S1D), which are highly correlated with immune processes [26] and tumor formation mechanisms [27, 28], as well as other diseases $[29,30]$.

Differential expression profiles of mRNAs in gastric cancer. The 4 mRNA microarray results are shown in Table S2.0. GSE2685 contains 15 pairs of samples; GSE103236 consists of 19 samples; GSE54129 contains 66 pairs of samples, and GSE79973 contains 10 pairs of samples. There were 5601, 6039, 12964 and 22187 mRNAs between GC and normal tissues, respectively, including 1201 shared mRNAs (Figure 2A). We selected the fourth microarray dataset as our study subject because the first and third microarray datasets could not be obtained from the GC and the adjacent tissues of
GC, and the second microarray dataset was missing a sample. To obtain genes associated with both miRNA and gastric cancer, we utilized hsa-miR-375, which was confirmed by TargetScan, to search for targeted mRNAs. The results are shown in Figure 2B and we ultimately obtained 27 mRNAs (Table S2.1).

Similarly, we performed hierarchical clustering map and volcano plot analysis for the $27 \mathrm{mRNAs}$ according to the data from the fourth microarray (Figures 2C, 2D, Table S2.2). Additionally, 3 mRNAs were differentially expressed between GC patients and controls with the same cut-off value. PAX6 $(\log \mathrm{FC}=1.4956081, \mathrm{p}=3.59 \mathrm{E}-03)$ was upregulated, KLF4 $(\operatorname{logFC}=-1.995832, \mathrm{p}=1.46 \mathrm{E}-04)$ and ASCL1 $(\log \mathrm{FC}=-3.375971, \quad \mathrm{p}=1.05 \mathrm{E}-03)$ were downregulated. Because miRNAs usually acts by suppressing gene expression $[8,31]$, we thought that PAX6 was related to hsa-miR375. Meanwhile, some studies have reported that PAX6 can suppress the tumorigenicity of glioblastoma multiforme cells [32]; more importantly, the hypermethylation of PAX6 was 
A

genes microarray 2 genes microarray 4

genes microarray 1 343 5815

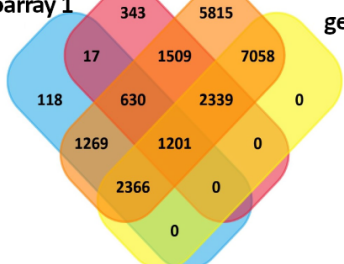

Benes sharing $_{\text {gen }}$

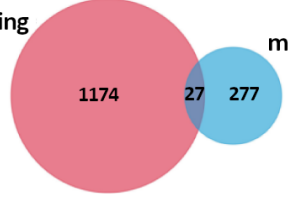

C

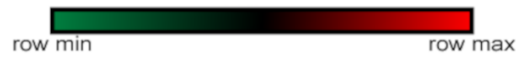

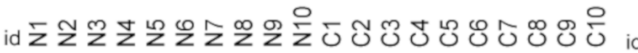

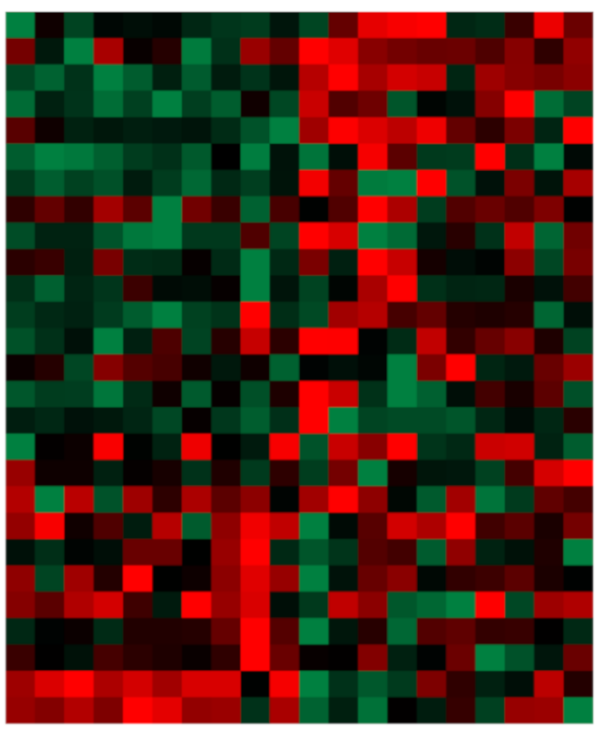

PAX6

$\mathrm{ABI} 2$

AGPS

$\mathrm{HSPH} 1$

YAP1

CCND2

GMFB

ELAVL4

CSNK2A1

DST

TCF4

SKI

YWHAB

$\mathrm{SHB}$

YWHAZ

PHLDA

PHLDA

POU3F1

SLC11A2
ARHGAP35

RGS16

SP1

BMPR2

INSR

KLF5

KIAA1549L

KLF4

ASCL1

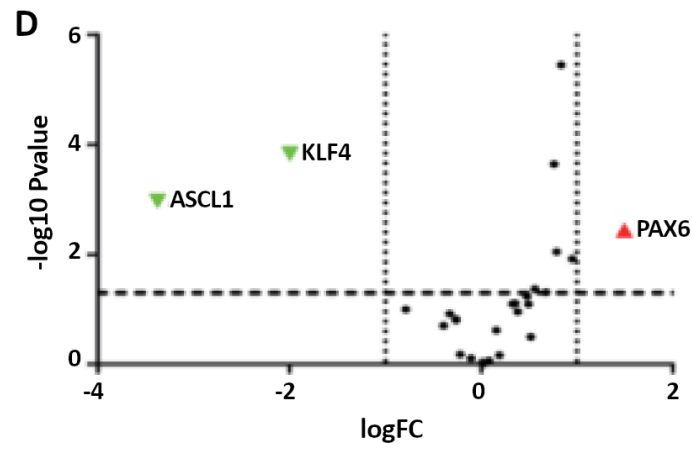

E

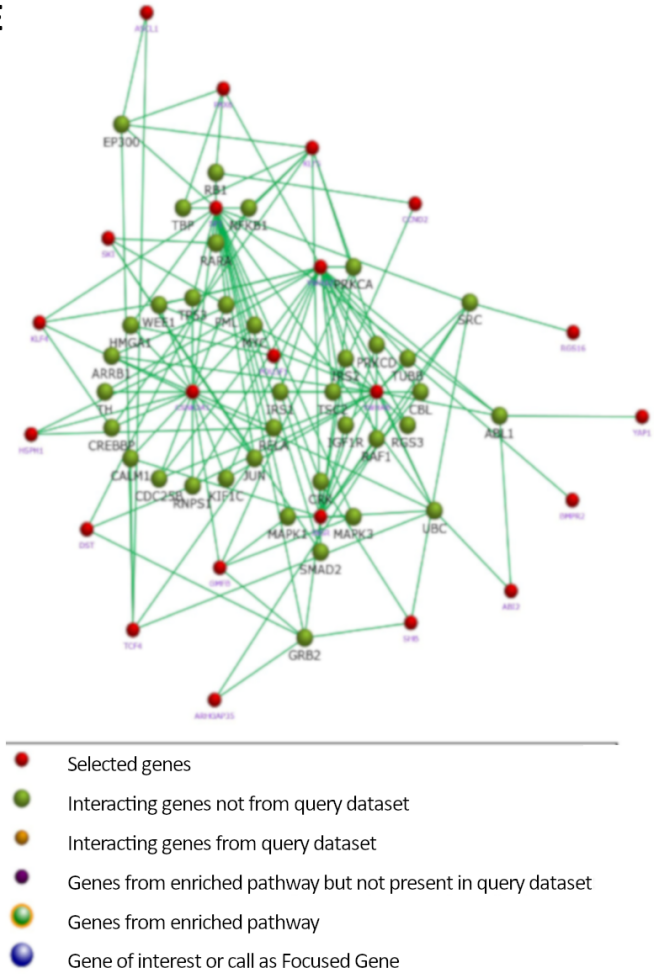

F

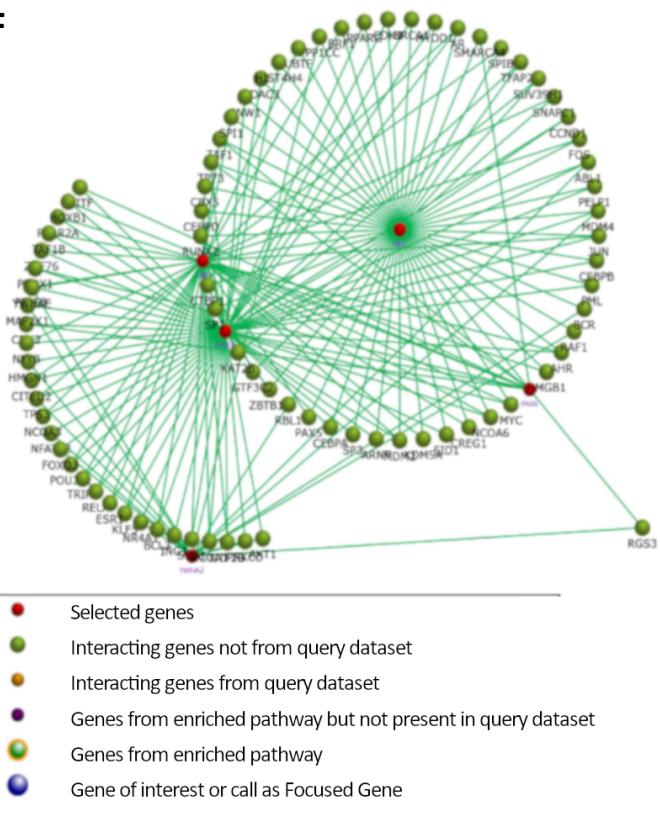

Figure 2. Expression profiles of mRNAs in gastric cancer. A) The number of mRNAs expressed between gastric cancer and normal tissues in 4 microarray results data with 1201 shared miRNAs. B) The number of shared mRNAs in the 4 microarrays that were targeted by hsa-miR-375 was 27 . C) Hierarchical clustering analysis indicated 27 mRNAs with differential expression profiles between GC (C1-C10) and adjacent tissues (N1-N10). The red and green shades represent expression levels above and below the expression among all samples, respectively (row min is 0 , row max is 1 ). $\mathrm{D}$ ) The differentially expressed mRNAs between gastric cancer and normal tissues in a volcano plot. The vertical lines correspond to a 2 -fold upregulation or downregulation, and the horizontal lines represent $\mathrm{p}=0.05$. The red points indicate the upregulated $\mathrm{mRNAs}$ and the green points indicate the downregulated mRNAs. E) The interaction of the 27 shared mRNAs with other genes. YWHAZ, EP300, TBP and RB1 were related to PAX6. F) The interaction of YWHAZ, EP300, TBP, RB1 and PAX6 with other genes. 
Table 1. Detailed information on the 3 differentially expressed circRNAs.

\begin{tabular}{|c|c|c|c|c|c|c|c|c|c|}
\hline \multirow{2}{*}{ organism } & \multirow{2}{*}{$\begin{array}{c}\text { position } \\
\text { (genome browser link) }\end{array}$} & \multirow{2}{*}{ strand } & \multirow{2}{*}{ circRNA ID } & \multirow{2}{*}{$\begin{array}{l}\text { genomic } \\
\text { length }\end{array}$} & \multirow{2}{*}{$\begin{array}{l}\text { spliced } \\
\text { length }\end{array}$} & \multirow{2}{*}{$\begin{array}{c}\text { best } \\
\text { transcript }\end{array}$} & \multirow{2}{*}{ gene symbol } & \multicolumn{2}{|c|}{ RNA-binding protein sites } \\
\hline & & & & & & & & junction & flanking regions \\
\hline hsa & chr6:157357968-157406039 & + & hsa_circ_0001658 & 48071 & 48071 & NM_017519 & ARID1B & EIF4A3 & $\begin{array}{l}\text { EIF4A3, FUS, PTB, } \\
\text { SFRS1, TIAL1 }\end{array}$ \\
\hline hsa & chr9:113703700-113773970 & - & hsa_circ_0004928 & 70270 & 39106 & NM_057159 & LPAR1 & - & EIF4A3 \\
\hline hsa & chr12:11199618-11248400 & - & hsa_circ_0000376 & 48782 & 48782 & NR_037918 & PRH1-PRR4 & - & $\begin{array}{c}\text { AUF1, EIF4A3, FUS, } \\
\text { HuR, QKI, U2AF65 }\end{array}$ \\
\hline
\end{tabular}

significantly associated with worse survival in GC patients [33], which confirmed our suspicions.

The interaction network of the shared 27 mRNAs and other genes revealed that YWHAZ, EP300, TBP and RB1 were related to PAX6 (Figure 2E). Interestingly, all of them directly correlated with GC, except TBP [34-37]. Therefore, we performed an interaction network plot (Figure 2F), which may have contributed to the exploration of new gene union pathways associated with gastric cancer that are different from those of the past.

Further analysis of PAX6 revealed that it mainly exists in signaling pathways regulating the pluripotency of stem cells and primarily affects the development of ectoderm according to DAVID Bioinformatics (Figure S2), which further extends our understanding of the mechanism of GC development.

Differential expression profiles of circRNAs in gastric cancer. Finally, we explored circRNAs and hsa-miR-375 in GC patients based on the above analysis. We obtained only 1 microarray dataset for circRNAs in GEO, as some studies did not submit their microarray results, and some samples were not obtained from patients (Table S3.0). Fifty-seven shared circRNAs were acquired from the microarray results (2902 circRNAs) and circRNAs targeted by hsa-miR-375 (622 circRNAs) through CircInteractome (Figure 3). The details of 57 circRNAs are shown in Table S3.1. We found that 3 circRNAs exhibited differential expression between GC and controls, with an absolute value of fold change (FC) of more than 2 and a p-value of less than 0.05 ; these circRNAs were hsa_circ_0001658 (FC=-3.63578, $\mathrm{p}=2.54 \mathrm{E}-05)$, hsa_circ_0004928 (FC=-3.00375, p=1.63E-02) and hsa circ_0000376 ( $\mathrm{FC}=2.17915, \mathrm{p}=1.64 \mathrm{E}-03)$. It is obvious that hsa_circ_0001658 and hsa_circ_0004928 were downregulated and that hsa_circ_0000376 was upregulated between the two groups. Given that circRNAs usually act as miRNA sponges to regulate gene expression and disease [38-42], we can obtain a preliminary conclusion that hsa_circ_0001658, hsa_circ_0004928 and hsa_circ_0000376 were significantly differentially expressed in GC patients, possibly resulting from hsa-miR-375.

We found that human PAX6 was targeted by hsa-miR375 through interactive analysis and TargetScan, hsa-miR375 mainly position $250-256$ of PAX6 3' UTR and in many species, both have the sequence conserved -GAACAAA(Figure S3); similarly, hsa_circ_0001658 ( $\mathrm{FC}=-3.63578$,

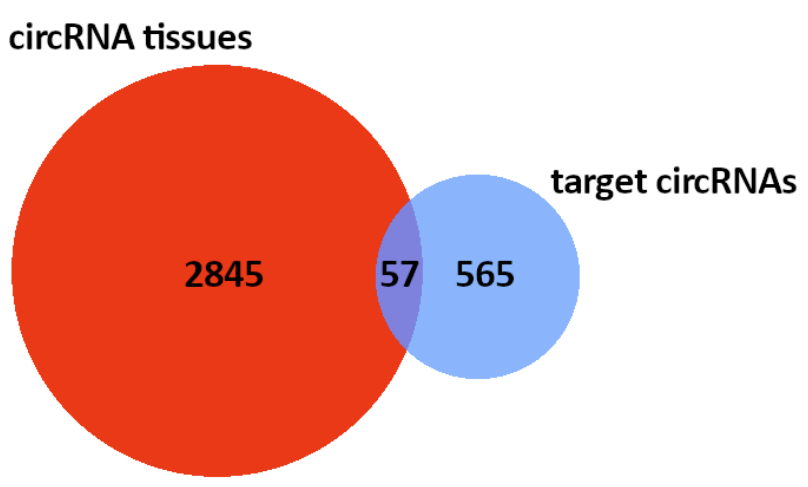

Figure 3. The number of circRNAs targeted by hsa-miR-375 in the microarray; there are 57 shared circRNAs among them.

$\mathrm{p}=2.54 \mathrm{E}-05)$, hsa_circ_0004928 $(\mathrm{FC}=-3.00375, \mathrm{p}=1.63 \mathrm{E}-02)$ and hsa_circ_0000376 (FC=2.17915, $\mathrm{p}=1.64 \mathrm{E}-03)$ were found to have a close relationship with hsa-miR-375 by the biological information analysis software CircInteractome (Figure 3, Table S3.1). Combined with current reports, we can speculate that the circ0001658/circ0004928/circ0000376miR-375-PAX6 axis exists in GC and is worth further investigation.

According to the existing research, we cannot ignore the fact that circRNAs can also regulate cancers by encoding proteins $[43,44]$, so we analyzed protein binding by hsa circ_0001658, hsa_circ_0004928 and hsa_circ_0000376, respectively, through circBase and CircInteractome (Table 1).

\section{Discussion}

The roles of miRNAs and circRNAs in carcinogenesis and cancer progression have attracted much attention because their expression, function and relationship in GC development are still largely elusive. In our present study, we obtained differentially expressed miRNAs, mRNAs and circRNAs between GC and matched non-tumor tissues from GEO and focused on the role of downregulated hsa-miR-375, upregulated PAX6 and circRNAs related to hsa-miR-375, as well as their underlying mechanism, in GC progression.

MiRNAs are short, single-stranded RNA molecules that regulate gene expression and are widely implicated in the pathogenesis of human diseases [7] Here, we found that 
6 miRNAs were dysregulated between GC patients and controls. Hsa-miR-1, hsa-miR-142-3p, hsa-miR-95, hsa-miR133a and hsa-miR-181d were increased but hsa-miR-375 was decreased in GC. We selected hsa-miR-375 as our further study subject because related reports have already been published on this miRNA. Hsa-miR-375 was mainly involved in the Glypican pathway and Wnt signaling pathway, both of which are correlated with cancer. However, we cannot exclude the possibility that the 5 upregulated miRNAs, or other deregulated miRNAs, also participate in the development of GC, even though there is limited microarray data. Therefore, dysregulated miRNAs in GC need further investigation, which is an issue for future studies.

Considering the relationship between miRNAs and mRNA, PAX6 was thought to interact with hsa-miR-375 in GC. A previously published study reported that the hypermethylation of PAX6 could lead to worse survival in GC [33]. In addition, genes associated with PAX6 are relevant to GC. Therefore, we speculate that hsa-miR-375 affects GC by contributing to PAX6 hypermethylation. There is no doubt that we need to perform additional experiments to verify these conclusions, which is a limitation of our present study that will be addressed in future work.

Next, we explored circRNAs, which have attracted the most attention recently. However, whether the formation mechanism or function pattern remains unclear. Based on the current evidence, circRNAs affect cancer cells by regulating the expression of oncogenic or tumor suppressive genes, depending on the type of cancer or even the stage [45]. However, miRNA sponges, circRNA-miRNA-mRNA axes and circRNA-miRNA-protein axes are the most frequently reported functional patterns for circRNAs [46, 47]. As circRNA HRCR-miR-223-ARC provided an attractive therapeutic target for the treatment of cardiac hypertrophy and heart failure [38], the circMTO1-miR-9-p21 axis is suggested as a potential target in hepatocellular carcinoma (HCC) treatment [39], cSMARCA5-miR-17-3p/miR-181b-5p-TIMP3 in HCC [40], circLARP4-miR-424-LATS1 interactions in GC [42], etc. With the most advanced analysis technology, we confirmed that the presence of hsa_circ_0001658, hsa circ_0004928 and hsa_circ_0000376 in GC was related to hsa-miR-375. Interestingly, PAX6 was inversely related to hsa-miR-375 in GC. Therefore, we speculate that circ0001658/ circ0004928/circ0000376-miR-375-PAX6 axis can modulate GC and that these 3 circRNAs may be potential biomarkers in GC, which is an interesting topic for further study.

In summary, we identified that hsa-miR-375 was decreased in GC and was related to increased PAX6, both of which were associated with GC. The regulatory network involving the circ0001658/circ0004928/circ0000376-miR-375-PAX6 axis may provide a new topic of investigation and a better understanding of GC.

Supplementary information is available in the online version of the paper.
Acknowledgements: We sincerely acknowledge our friends Chuang Chen and Lanfang Hong for their technical support and guidance. We are also grateful to the National Natural Science Foundation of China (81472940) for their grants and support.

\section{References}

[1] TORRE LA, BRAY F, SIEGEL RL, FERLAY J, LORTETTIEULENT J et al. Global cancer statistics, 2012. CA Cancer J Clin 2015; 65: 87-108. https://doi.org/10.3322/ caac. 2126

[2] FERLAY J, SOERJOMATARAM I, DIKSHIT R, ESER $\mathrm{S}$, MATHERS $\mathrm{C}$ et al. Cancer incidence and mortality worldwide: Sources, methods and major patterns in GLOBOCAN 2012. Int J Cancer 2015; 136: E359-86. https://doi. org/10.1002/ijc.29210

[3] PARKIN DM. The global health burden of infection-associated cancers in the year 2002. Int J Cancer 2006; 118: 3030 3044. https://doi.org/10.1002/ijc.21731

[4] DANG Y, OUYANG X, ZHANG F, WANG K, LIN Y et al. Circular RNAs expression profiles in human gastric cancer. Sci Rep 2017; 7: 9060. https://doi.org/10.1038/s41598-01709076-6

[5] LIU X, CAI H, WANG Y. Prognostic significance of tumour markers in Chinese patients with gastric cancer. ANZ J Surg 2014; 84: 448-453. https://doi.org/10.1111/j.14452197.2012.06287.x

[6] SHAH MY, FERRAJOLI A, SOOD AK, LOPEZ-BERESTEIN G, CALIN GA. microRNA Therapeutics in Cancer - An Emerging Concept. EBioMedicine 2016; 12: 34-42. https:// doi.org/10.1016/j.ebiom.2016.09.017

[7] BERINDAN-NEAGOE I, MONROIG PDEL C, PASCULLI B, CALIN GA. MicroRNAome genome: A treasure for cancer diagnosis and therapy. CA Cancer J Clin 2014; 64: 311336. https://doi.org/10.3322/caac.21244

[8] BARTEL DP. MicroRNAs: Genomics, Biogenesis, Mechanism, and Function. Cell 2004; 116: 281-297. https://doi. org/10.1016/S0092-8674(04)00045-5

[9] MENDELL JT, OLSON EN. MicroRNAs in stress signaling and human disease. Cell 2012; 148: 1172-1187. https://doi. org/10.1016/j.cell.2012.02.005

[10] SAITO M, SCHETTER AJ, MOLLERUP S, KOHNO T, SKAUG $\mathrm{V}$ et al. The association of microRNA expression with prognosis and progression in early-stage, non-small cell lung adenocarcinoma: a retrospective analysis of three cohorts. Clin Cancer Res 2011; 17: 1875-1882. https://doi. org/10.1158/1078-0432.CCR-10-2961

[11] SCHETTER AJ, LEUNG SY, SOHN JJ, ZANETTI KA, BOWMAN ED et al. MicroRNA expression profiles associated with prognosis and therapeutic outcome in colon adenocarcinoma. JAMA 2008; 299: 425-436. https://doi. org/10.1001/jama.299.4.425

[12] CHAN M, LIAW CS, JI SM, TAN HH, WONG CY et al. Identification of circulating microRNA signatures for breast cancer detection. Clin Cancer Res 2013; 19: 4477-4487. https://doi.org/10.1158/1078-0432.CCR-12-3401 
[13] MARCHINI S, CAVALIERI D, FRUSCIO R, CALURA E, GARAVAGLIA D et al. Association between miR-200c and the survival of patients with stage I epithelial ovarian cancer: a retrospective study of two independent tumour tissue collections. Lancet Oncol 2011; 12: 273-285. https://doi. org/10.1016/S1470-2045(11)70012-2

[14] ZHANG X, YAN Z, ZHANG J, GONG L, LI W et al. Combination of hsa-miR-375 and hsa-miR-142-5p as a predictor for recurrence risk in gastric cancer patients following surgical resection. Ann Oncol 2011; 22: 2257-2266. https://doi. org/10.1093/annonc/mdq758

[15] PETKOVIC S, MULLER S. RNA circularization strategies in vivo and in vitro. Nucleic Acids Res 2015; 43: 2454-2465. https://doi.org/10.1093/nar/gkv045

[16] LI J, YANG J, ZHOU P, LE Y, ZHOU C et al. Circular RNAs in cancer: novel insights into origins, properties, functions and implications. Am J Cancer Res 2015; 5: 472-480.

[17] MENG X, LI X, ZHANG P, WANG J, ZHOU Y et al. Circular RNA: an emerging key player in RNA world. Brief Bioinform 2017; 18: 547-557. https://doi.org/10.1093/bib/bbw045

[18] LI P, CHEN S, CHEN H, MO X, LI T et al. Using circular RNA as a novel type of biomarker in the screening of gastric cancer. Clin Chim Acta 2015; 444: 132-136. https://doi. org/10.1016/j.cca.2015.02.018

[19] THE GENE ONTOLOGY CONSORTIUM. Expansion of the Gene Ontology knowledgebase and resources. Nucleic Acids Res 2017; 45: D331-D338. https://doi.org/10.1093/ nar/gkw1108

[20] KHATRI P, SIROTA M, BUTTE AJ. Ten Years of Pathway Analysis: Current Approaches and Outstanding Challenges. PLoS Comput Biol 2012; 8: e1002375. https://doi. org/10.1371/journal.pcbi.1002375

[21] WEBER D, AMAR L, GODDE D, PRINZ C. Extensive screening of microRNA populations identifies hsa-miR-375 and hsa-miR-133a-3p as selective markers for human rectal and colon cancer. Oncotarget 2018; 9: 27256-27267. https:// doi.org/10.18632/oncotarget.25535

[22] BIERKENS M, KRIJGSMAN O, WILTING SM, BOSCH L, JASPERS A et al. Focal aberrations indicate EYA2 and hsamiR-37 as oncogene and tumor suppressor in cervical carcinogenesis. Genes Chromosomes Cancer 2013; 52: 56-68. https://doi.org/10.1002/gcc.22006

[23] ZEHENTMAYR F, HAUSER-KRONBERGER C, ZELLINGER B, HLUBEK F, SCHUSTER C et al. Hsa-miR-375 is a predictor of local control in early stage breast cancer. Clin Epigenetics 2016; 8: 28. https://doi.org/10.1186/s13148-0160198-1

[24] GIRICZ O1, REYNOLDS PA, RAMNAUTH A, LIU C, WANG T et al. Hsa-miR-375 is differentially expressed during breast lobular neoplasia and promotes loss of mammary acinar polarity. J Pathol 2012; 226: 108-119. https://doi. org/10.1002/path.2978

[25] BLECK B, GRUNIG G, CHIU A, LIU M, GORDON T et al. MicroRNA-375 regulation of thymic stromal lymphopoietin by diesel exhaust particles and ambient particulate matter in human bronchial epithelial cells. J Immunol 2013; 190: 3757-3763. https://doi.org/10.4049/jimmunol.1201165
[26] LI N, FU H, HEWITT SM, DIMITROV DS, HO M. Therapeutically targeting glypican-2 via single-domain antibodybased chimeric antigen receptors and immunotoxins in neuroblastoma. Proc Natl Acad Sci U S A 2017; 114: E6623E6631. https://doi.org/10.1073/pnas.1706055114

[27] QADIR MI, PARVEEN A, ALI M. Cdc42: Role in Cancer Management. Chem Biol Drug Des 2015; 86: 432-439. https://doi.org/10.1111/cbdd.12556

[28] ZENG X, ZHANG Y, XU H, ZHANG T, XUE Y et al. Secreted Frizzled Related Protein 2 Modulates Epithelial-Mesenchymal Transition and Stemness via Wnt/ $\beta$-Catenin Signaling in Choriocarcinoma. Cell Physiol Biochem 2018; 50: 1815-1831. https://doi.org/10.1159/000494862

[29] MATTHIJS BLANKESTEIJN W, HERMANS KC. Wnt signaling in atherosclerosis. Eur J Pharmacol 2015; 763: 122130. https://doi.org/10.1016/j.ejphar.2015.05.023

[30] CLEVERS H, NUSSE R. Wnt/b-Catenin Signaling and Disease. Cell 2012; 149: 1192-1205. https://doi.org/10.1016/j. cell.2012.05.012

[31] GUO L, ZHAO Y, YANG S, ZHANG H, CHEN F. Integrative Analysis of miRNA-mRNA and miRNA-miRNA Interactions. Biomed Res Int 2014; 2014: 907420. https://doi. org/10.1155/2014/907420

[32] MAYES DA, HU Y, TENG Y, SIEGEL E, WU X et al. PAX6 Suppresses the Invasiveness of Glioblastoma Cells and the Expression of the Matrix Metalloproteinase-2 Gene. Cancer Res 2006; 66: 9809-9817. https://doi.org/10.1158/0008-5472. CAN-05-3877

[33] YANG Q, SHAO Y, SHI J, QU Y, WU K et al. Concomitant PIK3CA amplification and RASSF1A or PAX6 hypermethylation predict worse survival in gastric cancer. Clin Biochem 2014; 47: 111-116. https://doi.org/10.1016/j.clinbiochem.2013.10.014

[34] VERMA N, HUNG KH, KANG JJ, BARAKAT NH, STUMPH WE. Differential Utilization of TATA Box-binding Protein (TBP) and TBP-related Factor 1 (TRF1) at Different Classes of RNA Polymerase III Promoters. J Biol Chem 2013; 288: 27564-27570. https://doi.org/10.1074/jbc.C113.503094

[35] BARGIELA-IPARRAGUIRRE J, PRADO-MARCHAL L, FERNANDEZ-FUENTE M, GUTIERREZ-GONZALEZ A, MORENO-RUBIO J et al. CHK1 expression in Gastric Cancer is modulated by $\mathrm{p} 53$ and $\mathrm{RB} 1 / \mathrm{E} 2 \mathrm{~F} 1$ : implications in chemo/radiotherapy response. Sci Rep 2016; 6: 21519. https://doi.org/10.1038/srep21519

[36] GUO F, GAO Y, SUI G, JIAO D, SUN L et al. miR-375-3p/ YWHAZ/ $\beta$-catenin axis regulates migration, invasion, EMT in gastric cancer cells. Clin Exp Pharmacol Physiol 2019; 46: 144-152. https://doi.org/10.1111/1440-1681.13047

[37] CAMPBELL IG, CHOONG D, CHENEVIX-TRENCH G. No germline mutations in the histone acetyltransferase gene EP300 in BRCA1 and BRCA2 negative families with breast cancer and gastric, pancreatic, or colorectal cancer. Breast CancerRes 2004; 6:R366-371.https://doi.org/10.1186/bcr803

[38] WANG K, LONG B, LIU F, WANG JX, LIU CY et al. A circular RNA protects the heart from pathological hypertrophy and heart failure by targeting miR-223. Eur Heart J 2016; 37 : 2602-2611. https://doi.org/10.1093/eurheartj/ehv713 
[39] HAN D, LI J, WANG H, SU X, HOU J et al. Circular RNA circMTO1 acts as the sponge of microRNA-9 to suppress hepatocellular carcinoma progression. Hepatology 2017; 66: 1151-1164. https://doi.org/10.1002/hep.29270

[40] YU J, XU QG, WANG ZG, YANG Y, ZHANG L et al. Circular RNA cSMARCA5 inhibits growth and metastasis in hepatocellular carcinoma. J Hepatol 2018; 68: 1214-1227. https://doi.org/10.1016/j.jhep.2018.01.012

[41] ZHENG Q, BAO C, GUO W, LI S, CHEN J et al. Circular RNA profiling reveals an abundant circHIPK3 that regulates cell growth by sponging multiple miRNAs. Nat Commun 2016; 7: 11215. https://doi.org/10.1038/ncomms11215

[42] ZHANG J, LIU H, HOU L, WANG G, ZHANG R et al. Circular RNA_LARP4 inhibits cell proliferation and invasion of gastric cancer by sponging miR-424-5p and regulating LATS1 expression. Mol Cancer 2017; 16: 151. https://doi. org/10.1186/s12943-017-0719-3

[43] YANG Y, GAO X, ZHANG M, YAN S, SUN C et al. Novel Role of FBXW7 Circular RNA in Repressing Glioma Tumorigenesis. J Natl Cancer Inst 2018; 110. https://doi. org/10.1093/jnci/djx166
[44] CONN SJ, PILLMAN KA, TOUBIA J, CONN VM, SALMANIDIS $\mathrm{M}$ et al. The RNA Binding Protein Quaking Regulates Formation of circRNAs. Cell 2015; 160: 11251134. https://doi.org/10.1016/j.cell.2015.02.014

[45] DU WW, YANG W, LIU E, YANG Z, DHALIWAL P et al. Foxo3 circular RNA retards cell cycle progression via forming ternary complexes with p21 and CDK2. Nucleic Acids Res 2016; 44: 2846-2858. https://doi.org/10.1093/nar/ gkw027

[46] MEMCZAK S, JENS M, ELEFSINIOTI A, TORTI F, KRUEGER J et al. Circular RNAs are a large class of animal RNAs with regulatory potency. Nature 2013; 495: 333-338. https://doi.org/10.1038/nature11928

[47] HANSEN TB, JENSEN TI, CLAUSEN BH, BRAMSEN JB, FINSEN B et al. Natural RNA circles function as efficient microRNA sponges. Nature 2013; 495: 384-388. https://doi. org/10.1038/nature11993 